\title{
Serum Neurofilament Light in Patients with Frontotemporal Dementia Caused by CHMP2B Mutation
}

\author{
Anders Toft ${ }^{a} \quad$ Peter Roos $^{a}$ Olli Jääskeläinen ${ }^{b} \quad$ Christian Sandøe Musaeus $^{a}$ \\ Emil Elbæk Henriksen ${ }^{a}$ Peter Johannsen ${ }^{c}$ Troels Tolstrup Nielsen ${ }^{a}$ \\ Sanna-Kaisa Herukka ${ }^{\text {b }}$ Anja Hviid Simonsen ${ }^{a}$ Jørgen Erik Nielsen ${ }^{a}$ \\ ${ }^{a}$ Neurogenetics Clinic \& Research Lab, Danish Dementia Research Centre, Copenhagen, Denmark; ${ }^{\mathrm{b}}$ Institute of \\ Clinical Medicine - Neurology, University of Eastern Finland, Ritva, Finland; ' ${ }^{2}$ Medical \& Science, Novo Nordisk A/S, \\ Søborg, Denmark
}

\section{Keywords \\ CHMP2B · Frontotemporal dementia · Neurofilament light · Presymptomatic}

\begin{abstract}
Introduction: The potential of neurofilament light (NfL) as a blood-based biomarker is currently being investigated in autosomal dominant neurodegenerative disease. This study explores the clinical utility of serum-NfL in frontotemporal dementia due to CHMP2B mutation (FTD-3). Methods: This cross-sectional study included serum and CSF data from 38 members of the Danish FTD-3 family: 12 affected $C H M P 2 B$ mutation carriers, 10 presymptomatic carriers, and 16 noncarriers. Serum-NfL levels measured by single-molecule array (Simoa) technology were tested for associations with the clinical groups and clinical parameters. Serum and CSF data were compared, and CSF/serum-albumin ratio was included as a measure of blood-brain barrier (BBB) function. Results: Serum-NfL concentrations were significantly increased in symptomatic CHMP2B mutation carriers compared to presymptomatic carriers and in both groups compared to healthy family controls. Serum-NfL levels appear to increase progressively with age in presymptomatic carriers, and this is per-
\end{abstract}

karger@karger.com

(C) 2021 S. Karger AG, Basel

www.karger.com/dem

Karger" haps followed by a change in trajectory when patients become symptomatic. Measurements of NfL in serum and CSF were highly correlated and fold-changes in serum and CSF between clinical groups were similar. Increase in serum-NFL levels was correlated with reduced ACE-score. Higher CSF/ serum-albumin ratios were demonstrated in FTD-3 patients, but this did not affect the significant associations between serum-NfL and clinical groups. Conclusion: Serum-NfL could be utilized as an accurate surrogate marker of CSF levels to segregate symptomatic CHMP2B carriers, presymptomatic carriers, and non-carriers. The observed indication of BBB dysfunction in FTD-3 patients did not confound this use of serum-NfL. The results support the occurrence of mutationrelated differences in NfL dynamics in familial FTD.

(c) 2021 S. Karger AG, Basel

\section{Introduction}

Frontotemporal dementia (FTD) comprises a group of neurodegenerative disorders, with clinical syndromes encompassing behavioral changes, language impairment, and general cognitive decline. Familial aggregation is frequent, and high-risk variants have presently been identi- 
fied in 11 genes [1]. In a large Danish family, a proteintruncating variant in the $C H M P 2 B$ gene $($ c.532-1G $>C$ ) on chromosome 3 was discovered as the cause of earlyonset autosomal dominant FTD, termed FTD-3 [2]. More than 50 members of this family have been diagnosed with clinical FTD-3, and a number of mutation carriers are presymptomatic. Studies indicate that the neuropathological process in genetic FTD is ongoing several years prior to symptom onset [3,4]. As early detection of developing pathology may hold significance for treatment timing in upcoming therapeutic trials, there is an unmet need for validated biomarkers to identify this preclinical stage.

One relevant biomarker in neurodegenerative disease is neurofilament light (NfL) protein; a low-molecularweight cytoskeletal subunit exclusively expressed in neurons [5]. Considered a sensitive and nonspecific biomarker of axonal damage, NfL has been shown to increase with age in healthy participants, and relatively higher CSF levels have segregated patients from healthy controls in a range of neurodegenerative disorders including ALS, Parkinson's disease, Alzheimer's disease, and vascular dementia [6]. A high correlation between CSF-NfL and the many fold lower concentration in serum has been reported for several of these conditions [7]. In genetic FTD caused by mutation in the GRN, C9orf72, or MAPT gene, serum-NfL was recently found to increase in presymptomatic individuals just prior to symptom onset and remain elevated in symptomatic patients compared to presymptomatic carriers and noncarriers [8]. Increase in serum-NfL was associated with reduced MiniMental State Examination (MMSE) score and gray matter atrophy, in line with findings from similar studies $[9,10]$. Interestingly, serum-NfL dynamics were shown to differ based on the type of FTD mutation [8].

In familial FTD due to $C H M P 2 B$ mutation, we have recently demonstrated significantly different levels of $\mathrm{NfL}$ in CSF from symptomatic carriers compared to presymptomatic carriers and in both groups compared to noncarrier family controls [4]. However, the capability of serum-NfL to separate clinical groups and its correlation with CSF levels and clinical parameters have not been investigated in FTD-3. Additionally, as we have previously observed reduced regional cerebral blood flow in presymptomatic $C H M P 2 B$ mutation carriers, as an indicator of potential capillary pathology [11], we included a measure of blood-brain barrier (BBB) dysfunction, to control for a possible confounder of an association between levels of NfL in serum and CSF. This study represents a crosssectional analysis of fluid biomarker data obtained from 38 members of the Danish FTD-3 family.

\section{Methods}

\section{Study Population}

For 6 generations, the Danish FTD-3 family has been studied through the FTD Research in Jutland Association (FReJA). Four hundred members are alive and at risk of harboring the pathogenic $C H M P 2 B$ variant. Diagnostic workup is performed at symptom onset, and if consented to, biofluid samples are stored in the Danish Dementia Biobank from clinically affected patients, presymptomatic carriers, and non-carrier family members.

This study included data from 38 individuals: 12 affected CHMP2B mutation carriers, 10 presymptomatic carriers, and 16 noncarriers. All participants were clinically evaluated at the time of sampling, and participant age was based on this time point. Disease duration was defined as the time from diagnosis to biofluid sampling. Addenbrooke's Cognitive Examination (ACE) score was employed as a measure of cognitive impairment.

\section{Laboratory Procedures}

CSF samples were collected by standardized lumbar puncture. CSF-NfL concentrations were analyzed by sandwich ELISA, as previously described [4]. Serum-NfL was measured according to the instructions of the manufacturer using the NF-Light Advantage Kit for the Quanterix single-molecule array (Simoa, Lexington, MA, USA) at the Institute of Clinical Medicine-Neurology, University of Eastern Finland. Measurements of albumin concentrations were performed on Cobas 8,000 instruments (Roche diagnostics) at the Department of Clinical Biochemistry, Rigshospitalet, using commercial kits.

\section{Statistics}

Data were analyzed using R, version 3.6.1 (R Foundation for Statistical Computing, Vienna, Austria). NfL and albumin values were logarithmically transformed to obtain normal distribution. For descriptive statistics, comparisons between groups were performed with Kruskal-Wallis test (untransformed data) and ANOVA with post hoc Tukey test (transformed data). Variables were subsequently analyzed by general linear modeling and multinomial logistic regression, adjusted for age and significant interactions. Correlations were calculated using Pearson's correlation coefficient (normally distributed data) or Spearman's Rank Correlation Coefficient (ACE-score data). $p$ values below 5\% were considered significant.

\section{Results}

CSF and serum data were available for 32 and 31 participants, respectively. Patient characteristics and distribution of fluid biomarker data are presented in Table 1.

Group demographics showed no difference in distribution of sex, whereas presymptomatic participants constituted a significantly younger age-group. ACE-scores were expectedly lower in clinically affected FTD-3 patients, while levels of CSF-NfL, serum-NfL, and albumin ratios were significantly higher (shown in Table 1). 
Table 1. Patients characteristics and levels of fluid biomarkers

\begin{tabular}{|c|c|c|c|c|}
\hline Clinical group & Noncarriers & $\begin{array}{l}\text { Presymptomatic } \\
C H M P 2 B \\
\text { mutation carriers }\end{array}$ & $\begin{array}{l}\text { Symptomatic } \\
C H M P 2 B \\
\text { mutation carriers }\end{array}$ & $p$ value \\
\hline Sex, females/males & \multirow[t]{2}{*}{$8 / 8$} & \multirow[t]{2}{*}{$6 / 4$} & \multirow[t]{2}{*}{$3 / 9$} & 0.267 \\
\hline Age, years, mean (range) & & & & 0.011 \\
\hline Symptomatic versus noncarriers & \multirow{3}{*}{$60.1(38.1-71.1)$} & \multirow{3}{*}{$50.5(32.7-68.1)$} & \multirow{3}{*}{$62.8(53.6-73.3)$} & 0.701 \\
\hline Presymptomatic versus noncarriers & & & & 0.029 \\
\hline ACE, mean (range) $(\mathrm{CI})$ & & & & $<0.001$ \\
\hline Symptomatic versus noncarriers & $93.6(87-100)$ & \multirow{4}{*}{$\begin{array}{l}90.4(81-96) \\
(86.9-94.0)\end{array}$} & \multirow{4}{*}{$\begin{array}{l}70.6(41.0-89) \\
(60.1-81.2)\end{array}$} & $<0.001$ \\
\hline Symptomatic versus presymptomatic & \multirow[t]{3}{*}{$(91.4-95.9)$} & & & $<0.001$ \\
\hline Presymptomatic versus noncarriers & & & & 0.715 \\
\hline CSF-NfL, pg/mL, mean (CI) & & & & $<0.001$ \\
\hline Symptomatic versus noncarriers & \multirow{4}{*}{$13.6(7.2-20.0)$} & \multirow{4}{*}{$26.8(16.9-36.7)$} & \multirow{4}{*}{$67.0(33.5-100.5)$} & $<0.001$ \\
\hline Symptomatic versus presymptomatic & & & & 0.015 \\
\hline Presymptomatic versus non-carriers & & & & 0.503 \\
\hline $\mathrm{CSF} /$ serum-albumin ratio $(\mathrm{CI})$ & & & & 0.054 \\
\hline Symptomatic versus noncarriers & \multirow{3}{*}{$5.2(4.1-6.3)$} & \multirow{3}{*}{$6.1(4.5-7.7)$} & \multirow{3}{*}{$8.2(6.1-10.2)$} & 0.020 \\
\hline Symptomatic versus presymptomatic & & & & 0.223 \\
\hline Presymptomatic versus noncarriers & & & & 0.742 \\
\hline
\end{tabular}

ACE, Addenbrooke’s Cognitive Examination; NfL, neurofilament light; CI 95\%, confidence interval.

In this cohort, mean concentration of $\mathrm{NfL}$ in serum (30.1 pg/mL, range $5.3-187.8 \mathrm{pg} / \mathrm{mL}$ ) was 54 times lower than the corresponding value in CSF $(1,654.8 \mathrm{pg} / \mathrm{mL}$, 273.4-4,009.9). FTD-3 patients had 2.4-fold and 4.7fold higher median serum-NfL than presymptomatic and healthy individuals, respectively, and between the latter groups, the fold change was 2.0 (for CSF-NfL the corresponding fold-changes were 1.7, 4.5, and 2.7). Measurements of NfL in CSF and serum were highly correlated $\left(R^{2}=0.76, p<0.001\right.$, Pearson's correlation coefficient $=0.87)$ (shown in Fig. 1). ACE-scores decreased with increasing serum-NfL levels (Spearman's correlation coefficient $=-0.70, p<0.0001$ ), also when controlling for age in multiple regressions $(p<0.001)$. In a general linear model adjusted for age, serum-NfL values differed between symptomatic and presymptomatic CHMP2B mutation carriers $(p=0.019)$; symptomatic and healthy participants $(p<0.001)$; as well as presymptomatic and healthy participants $(p=0.040)$ (shown in Fig. 1). This model included age $(p=0.052)$ and a significant interaction between the effect of clinical status and age on NfL.

Serum Neurofilament Light in CHMP2B-Mediated FTD
In clinically affected FTD-3 patients, neither serumNfL nor albumin ratio was significantly associated with disease duration. For the entire population, serum-NfL levels were positively correlated with increasing albumin ratio $(p=0.021)$. When adding the albumin ratio to the general linear model, the statistical significance of the group differences in serum-NfL persisted, except for the comparison between presymptomatic and healthy participants $(p=0.079)$. In a multinomial logistic regression analysis including the same factors (with clinical group as a 3-level outcome variable), all groups differed significantly from 1 another. This was also the case when separating groups based on CSF-NfL instead of serum-NfL in an otherwise similar linear regression model.

\section{Discussion}

As focus has shifted towards identification of more readily accessible, blood-based biomarkers, this study explores the clinical utility of serum-NfL in familial FTD caused by $C H M P 2 B$ mutation. CSF and serum data from 


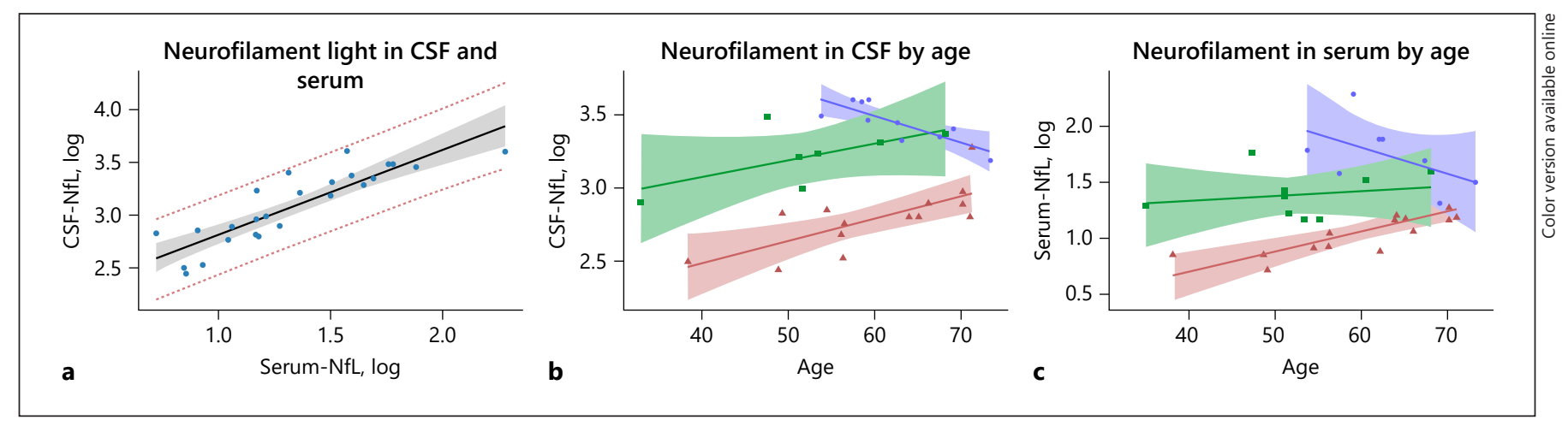

Fig. 1. CSF-serum correlation and NfL levels in CSF and serum by age. a depicts the linear relationship between measurements of neurofilament light protein in CSF and serum. 95\% confidence interval and prediction limits are shown. $\mathbf{b}$ and $\mathbf{c}$ represent generalized linear models of associations between age and NfL level in symptomatic (blue), presymptomatic (green) and non-carrier (red) participants. Neurofilament data are logarithmically transformed.

12 clinically affected patients, 10 presymptomatic CHMP2B mutation carriers, and 16 noncarriers from the Danish FTD-3 family were included.

Serum-NfL levels were highly correlated with the 54fold higher CSF-values. Fold-changes between the clinical groups were similar for serum and CSF. Based on serum-NfL concentrations, FTD-3 patients could be separated from presymptomatic carriers, and both groups from noncarrier family controls. These differences remained statistically significant when adjusted for age. Elevated serum-NfL levels were detectable several years before expected age of symptom onset (shown in Fig. 1), plausibly reflecting the early neurodegenerative process. In line with this, previous studies have demonstrated pathologic rates of cerebral atrophy in presymptomatic $C H M P 2 B$ mutation carriers $[12,13]$, but whether this is preceded by or coincides with the changes in serum-NfL remains to be investigated. In familial Alzheimer's disease, progressively rising serum-NfL has been observed more than a decade before symptom onset, prior to incipient changes in structural imaging and cognition [14, 15]. A recent cross-sectional study by the GENFI consortium in FTD due to mutation in the C9orf72, MAPT, or $G R N$ gene showed that accelerated brain atrophy and certain neuropsychological deficits were present 10 and 5 years before expected symptom onset, respectively [3]. This led to the prediction that serum-NfL levels would start to increase around 10 years before age of onset [10], but in a subsequent longitudinal study, presymptomatic mutation carriers grouped with the healthy controls in terms of serum-NfL level, and only symptomatic FTD patients had significantly increased values [8]. Post hoc an- alyzes, however, showed that presymptomatic individuals who became symptomatic within 1-2 years had higher baseline serum-NfL than those not approaching conversion. An unaffected level of NfL in presymptomatic participants or an abrupt increase immediately preceding the symptomatic stage has also been observed in similar studies $[9,14,16]$. Thus, it is still unclear whether $\mathrm{NfL}$ is a marker of imminent phenoconversion or if it does reflect the onset and progression of neurodegeneration over several years. The latter capability would seem to best enable the proposed future use of serum-NfL as a timing marker of potential disease-modifying treatment in the prodromal stage before neuropathology becomes too manifest. As the dynamics of NfL in this regard appear to differ among neurodegenerative disease entities as well as FTD subtypes, the biomarker may only prove useful in some of these disorders. To accurately determine the preclinical course of NfL in genetic subtypes of FTD, additional longitudinal studies with serial measurements of serum-NfL are needed. This is also a prerequisite for the potential use of serum-NfL as a basis of estimating individual time points of phenoconversion in presymptomatic subjects.

The depiction of our serum-NfL data as a function of age gives the impression that the increase in presymptomatic carriers is followed by a decrease in symptomatic patients (shown in Fig. 1). This subsequent decline or stabilization, which may perhaps be explained by protein aggregation or the presence of autoantibodies against NfL [17], has also been noted in FTD patients with C9orf72 or MAPT mutation - whereas levels in GRN mutation carriers have been found to persistently rise or fluctuate, per- 
haps due to a higher degree of neuroinflammation affecting rates of axonal degeneration [8]. Clarification of disorder-specific trajectories and variability of $\mathrm{NfL}$ in the symptomatic phase is important for it to function as a surrogate marker of treatment effect - another potential of the biomarker currently being studied. In multiple sclerosis, reductions in serum-NfL have been demonstrated after administration of disease-modifying therapies [18, 19]. Naturally, the use of serum-NfL as a pharmacodynamic biomarker in clinical trials is dependent on correlation with clinical parameters. In this study, we found that increasing serum-NfL levels were associated with decline in ACE-score, independent of age. Similarly, increasing levels or rates of change have been shown to correlate with MMSE-score, disease severity, brain atrophy rates, and survival in FTD $[8-10,16]$. Numerous studies have evaluated the diagnostic performance of serum-NfL: significant differences have been found among neurodegenerative disease categories - such as higher serum-NfL in FTD than Alzheimer's disease [20] - as well as between genetic FTD subtypes [8]. Nevertheless, this usage of serum-NfL is clearly limited by its nonspecificity, why genetic cohorts with known pathogenicity provide more suitable target groups, in the presymptomatic stage. Another limitation is the lack of knowledge about which confounding factors, in addition to sex, age, and neurologic comorbidity, that cause the intra- and interindividual variability in serumNfL. Furthermore, how the NfL protein is transported from CNS to the bloodstream is undetermined. One mechanism that has been discussed in particular is leakage across the BBB. In presymptomatic FTD-3 family members, we have previously observed reduced regional cerebral blood flow compared to noncarriers, on contrast-enhanced MRI [11]. The spin-echo sequences utilized were sensitive to signal from the capillaries, indicating involvement of capillaries in the pathophysiology of FTD-3. For this reason, we included a well-established measure of $\mathrm{BBB}$ integrity, namely $\mathrm{CSF} /$ serum-albumin ratio, to control for a potential confounder of the association between NfL levels in serum and CSF. The analyses showed higher albumin ratio in $C H M P 2 B$ mutation carriers than noncarriers (not shown), as well as in FTD-3 patients than healthy family controls, while statistical significance was not reached for comparisons with presymptomatic carriers. In a recent study of BBB integrity in dementias of different etiologies, we did not find the albumin ratio to be elevated in patients with (all-cause) FTD or Alzheimer's disease [21], suggesting that the potential BBB dysfunction is related to the $C H M P 2 B$ mutation. This should be investigated specifically, with techniques such as dynamic con- trast-enhanced MRI. However, when controlling the association between serum-NfL and clinical status for differing albumin ratios, it did not confound the significant correlations. This is in line with a recent meta-analysis in Alzheimer's disease [22], where several studies have found elevated serum-NfL levels in the absence of significantly increased albumin ratios. This indicates that transport of NfL to the bloodstream occurs via different pathways, which should be investigated further, as the concentration in serum may be confounded by varying degrees of release from the CNS [23].

Collectively, it is shown that serum-NfL can be used as a surrogate marker of CSF levels to trace progressive neurodegeneration years before expected debut of symptoms in FTD-3. Serum-NfL levels could effectively separate symptomatic $C H M P 2 B$ mutation carriers, presymptomatic carriers, and noncarriers. An elevated CSF/serum-albumin ratio was found in symptomatic FTD-3 patients as an indication of BBB dysfunction. This finding, however, did not confound the associations between NfL and clinical groups. The apparent linear course of serum-NfL in FTD caused by CHMP2B mutation supports the occurrence of gene-specific differences in NfL dynamics in familial FTD. Followup studies will determine if conversion to the symptomatic stage does in fact change the trajectory of serum-NfL and if the rate of change is a better performing biomarker than the absolute levels in this disorder.

Published in Celebration of the 30th Anniversary of the inception of Dementia and Geriatric Cognitive Disorders 1990-2020.

\section{Acknowledgements}

The Danish FTD-3 family members are greatly acknowledged for their dedication and contribution, which has been facilitated for more than 3 decades via the FReJA consortium.

\section{Statement of Ethics}

This study was approved by the Ethics Committee of the Capital Region of Denmark (H-1-2012-041). All participants provided written informed consent. Clinically affected FTD-3 patients were included in early disease stages to ensure legitimate informed consent.

\section{Conflict of Interest Statement}

The authors have no conflicts of interest to declare. 


\section{Funding Sources}

This study has been granted financial support by Aase and Ejnar Danielsens Foundation and the Novo Nordisk Foundation. The foundations were not involved in the preparation of data or the manuscript.

\section{Author Contributions}

Anders Toft contributed to study design, analysis and interpretation of data, drafting of the manuscript, and obtaining funding; Peter Roos contributed to study design, acquisition of data, analysis and interpretation of data, and revision of the manuscript for intellectual content; Olli Jääskeläinen contributed to acquisition of data and revision of the manuscript for intellectual content; Christian Sandøe Musaeus contributed to acquisition of data, analysis and interpretation of data and revision of the manuscript for intellectual content; Emil Elbæk Henriksen contributed to revision of the manuscript for intellectual content; Peter Johannsen contributed to revision of the manuscript for intellectual content; Troels Tolstrup Nielsen contributed to revision of the manuscript for intellectual content; Sanna-Kaisa Herukka contributed to acquisition of data and revision of the manuscript for intellectual content; Anja Hviid Simonsen contributed to revision of the manuscript for intellectual content; Jørgen Erik Nielsen contributed to study design, interpretation of data, revision of the manuscript for intellectual content, and obtaining funding.

\section{References}

1 Pottier C, Ravenscroft TA, Sanchez-Contreras M, Rademakers R. Genetics of FTLD: overview and what else we can expect form genetic studies. J Neurochem. 2016 Aug; 138(Suppl 1):32-53.

2 Skibinski G, Parkinson NJ, Brown JM, Chakrabarti L, Lloyd SL, Hummerich H, et al. Mutations in the endosomal ESCRTIII-complex subunit CHMP2B in frontotemporal dementia. Nat Genet. 2005 Aug;37(8):806-8.

3 Rohrer JD, Nicholas JM, Cash DM, van Swieten J, Dopper E, Jiskoot L, et al. Presymptomatic cognitive and neuroanatomical changes in genetic frontotemporal dementia in the genetic frontotemporal dementia initiative (GENFI) study: a cross-sectional analysis. Lancet Neurol. 2015 Mar;14(3):253-62.

4 Rostgaard N, Roos P, Portelius E, Blennow K, Zetterberg H, Simonsen AH, et al. CSF neurofilament light concentration is increased in presymptomatic CHMP2B mutation carriers. Neurology. 2018 Jan 9;90(2):e157-63.

5 Petzold A. Neurofilament phosphoforms: surrogate markers for axonal injury, degeneration and loss. J Neurol Sci. 2005 Jun 15; 233(1-2):183-98.

6 Bridel C, van Wieringen WN, Zetterberg $\mathrm{H}$, Tijms BM, Teunissen CE; the NFL Group, et al. Diagnostic value of cerebrospinal fluid neurofilament light protein in neurology: a systematic review and meta-analysis. JAMA Neurol. 2019 Jun 17;76(9):1035-48.

7 Khalil M, Teunissen CE, Otto M, Piehl F, Sormani MP, Gattringer T, et al. Neurofilaments as biomarkers in neurological disorders. Nat Rev Neurol. 2018 Oct;14(10):577-89.

8 van der Ende EL, Meeter LH, Poos JM, Panman JL, Jiskoot LC, Dopper EGP, et al. Serum neurofilament light chain in genetic frontotemporal dementia: a longitudinal, multicentre cohort study. Lancet Neurol. 2019 Dec; 18(12):1103-11.
9 Meeter LH, Dopper EG, Jiskoot LC, SanchezValle R, Graff C, Benussi L, et al. Neurofilament light chain: a biomarker for genetic frontotemporal dementia. Ann Clin Transl Neurol. 2016 Jul 1;3(8):623-36.

10 Rohrer JD, Woollacott IO, Dick KM, Brotherhood E, Gordon E, Fellows A, et al. Serum neurofilament light chain protein is a measure of disease intensity in frontotemporal dementia. Neurology. 2016 Sep 27;87(13):132936.

11 Lunau L, Mouridsen K, Rodell A, Ostergaard L, Nielsen JE, Isaacs A, et al. Presymptomatic cerebral blood flow changes in CHMP2B mutation carriers of familial frontotemporal dementia (FTD-3), measured with MRI. BMJ Open. 2012 Mar 15;2(2):e000368.

12 Eskildsen SF, Østergaard LR, Rodell AB, Østergaard L, Nielsen JE, Isaacs AM, et al. Cortical volumes and atrophy rates in FTD-3 CHMP2B mutation carriers and related noncarriers. Neuroimage. 2009 Apr 15;45(3): 713-21.

13 Rohrer JD, Ahsan RL, Isaacs AM, Nielsen JE, Ostergaard L, Scahill R, et al. Presymptomatic generalized brain atrophy in frontotemporal dementia caused by CHMP2B mutation. Dement Geriatr Cogn Disord. 2009;27(2):182-6.

14 Weston PSJ, Poole T, O'Connor A, Heslegrave A, Ryan NS, Liang Y, et al. Longitudinal measurement of serum neurofilament light in presymptomatic familial Alzheimer's disease. Alz Res Therapy. 2019 Feb 20;11(1):19.

15 Preische O, Schultz SA, Apel A, Kuhle J, Kaeser SA, Barro C, et al. Serum neurofilament dynamics predicts neurodegeneration and clinical progression in presymptomatic Alzheimer's disease. Nat Med. 2019 Feb;25(2): $277-83$.
16 Scherling CS, Hall T, Berisha F, Klepac K, Karydas A, Coppola G, et al. Cerebrospinal fluid neurofilament concentration reflects disease severity in frontotemporal degeneration. Ann Neurol. 2014 Jan;75(1):116-26.

17 Fialová L, Svarcová J, Bartos A, Ridzon P, Malbohan I, Keller O, et al. Cerebrospinal fluid and serum antibodies against neurofilaments in patients with amyotrophic lateral sclerosis. Eur J Neurol. 2010 Apr;17(4):562-6.

18 Disanto G, Barro C, Benkert P, Naegelin Y, Schädelin S, Giardiello A, et al. Serum neurofilament light: a biomarker of neuronal damage in multiple sclerosis. Ann Neurol. 2017 Jun;81(6):857-70.

19 Piehl F, Kockum I, Khademi M, Blennow K, Lycke J, Zetterberg $\mathrm{H}$, et al. Plasma neurofilament light chain levels in patients with MS switching from injectable therapies to fingolimod. Mult Scler. 2018 Jul;24(8):1046-54.

20 Forgrave LM, Ma M, Best JR, DeMarco ML. The diagnostic performance of neurofilament light chain in CSF and blood for Alzheimer's disease, frontotemporal dementia, and amyotrophic lateral sclerosis: a systematic review and meta-analysis. Alzheimers Dement. 2019 Nov 4;11:730-43.

21 Musaeus CS, Gleerup HS, Høgh P, Waldemar G, Hasselbalch SG, Simonsen AH. Cerebrospinal fluid/plasma albumin ratio as a biomarker for blood-brain barrier impairment across neurodegenerative dementias. J Alzheimers Dis. 2020 May;75(2):429-36.

22 Olsson B, Lautner R, Andreasson U, Öhrfelt A, Portelius E, Bjerke M, et al. CSF and blood biomarkers for the diagnosis of Alzheimer's disease: a systematic review and meta-analysis. Lancet Neurol. 2016 Jun;15(7):673-84.

23 Kalm M, Boström M, Sandelius Å, Eriksson Y, Ek CJ, Blennow K, et al. Serum concentrations of the axonal injury marker neurofilament light protein are not influenced by blood-brain barrier permeability. Brain Res. 2017 Aug 1;1668:12-9. 